\title{
Warum Naturalismen?
}

Historisches Problem und methodische Herausforderung

Entgegen aller vermeintlichen Evidenzen ist Naturalismus in den Künsten ein auf besondere Weise mehrdimensionales und spannungsreiches Thema. Die Kunstgeschichte vor allem der ersten Hälfte des 20. Jahrhunderts ist überaus reich an Anhaltspunkten hierfür und eine Reihe an Fragen, um die es dabei ging, beschäftigen die jüngere Forschung erneut; auch wenn die Perspektiven heute andere sind und der Begriff, Naturalismus' häufig vermieden wird. Ein prominentes Beispiel für dessen einstige Attraktivität ist Max Dvořáks Aufsatz Idealismus und Naturalismus in der gotischen Skulptur und Malerei von $1924 .{ }^{1}$ Angesichts der hier aufgerufenen konventionellen Opposition zwischen einer Sphäre des Geisteigen und der Ideen versus der wahrnehmbaren Welt als (alleiniger) Vorgabe und Maßstab entwickelt der Text eine bemerkenswerte Synthese. In wenigen Zügen entwirft er eine Konstellation, in der im hohen Mittelalter aus einer zutiefst spirituell geprägten Philosophie und Religiosität heraus der individuellen Erfahrung eine zunehmend größere Bedeutung zugekommen sei. Vor diesem Hintergrund wurden dann, zunächst in der Baukunst, die Beziehungen zwischen Raum und Gegenstand als ästhetisches Gefüge neu organisiert. Und im Anschluss daran sei in einem umfassenden Sinne künstlerische Arbeit und komplementär dieErfahrung von Kunstwerken-als besondere Form der Vergeistigung des Materiellen entfaltet worden. Dieser Prozess ist bei Dvořák der Kern eines „gotischen Naturalismus", der in seiner Bindung an die vielfältigsten Erscheinungen der Welt eine „geistige Belebung der Kunst“ impliziert sowie in kaum dagewesener Weise Vorstellungskraft und Phantasie freigesetzt und herausgefordert habe. ${ }^{2}$

Es ist kaum zu übersehen, dass sich diese Überlegungen in den Grundriss einer Kunstgeschichte unter idealistischen Vorzeichen fügen, mitsamt ihren unaufhebbaren Dichotomien. In diesem Gefüge jedoch steht Naturalismus keineswegs bloß für die eine

1 Max Dvořák: Idealismus und Naturalismus in der gotischen Skulptur und Malerei, in: ders.: Kunstgeschichte als Geistesgeschichte. Studien zur abendländischen Kunstentwicklung, München 1924, S. 41-147; im Folgenden nach der Ausgabe Max Dvořák: Studien zur Kunstgeschichte, hg. von Irma Emmrich, Leipzig 1989, S. 160-268.

2 Dvořák 1989 (wie Anm. 1), S. 215 und 229f. 
von zwei unvereinbaren Seiten, sondern für deren durch subjektive Erfahrung und künstlerische Arbeit hergestellte dialektische Verschränkung. Diese Beobachtung bildet den gedanklichen Ausgangspunkt dieses Sammelbandes. - Vergleichbar, aber weitaus grundlegender und in einer allgemein systematischen Perspektive hatte Alois Riegl bereits um 1900 einen „naturalistischen 'Trieb“ als eine wichtige Komponente des menschlichen Kunstwollens generell verstanden. ${ }^{3}$ Er tendiere - so Riegl - zu optisch fernsichtigen Bildkonzepten und Stilmerkmalen, und er habe die kulturelle Funktion einer sukzessiven Klärung der anfangs „nebelhaften Erinnerungsbilder“ der Menschen von den „Naturwerken“ gehabt. Im Anschluss an Riegl - und damit sei eine weitere Facette von Naturalismus lediglich angerissen - akzentuierte Wilhelm Worringer eine vor allem psychologischemotionale Dimension. In naturalistischen Strömungen der Kunst zeige sich ihm zufolge vor allem ein Bedürfnis nach „Einfühlung“, die zusammen mit ihrem Gegenpart - der „Abstraktion“ - als eine jener beiden Facetten eines allgemein menschlichen „Selbstentäußerungsdrangs“ zu verstehen sei, die als polare Gegensätze jedes „Kunstwollen“ antreiben. In dieser Konstellation wird „Naturalismus“ grundsätzlich vom Anliegen bloßer Imitation der Körper und Erscheinungen unterschieden. ${ }^{4}$ Er fasst vielmehr alle Elemente eines Kunstwerkes, „die aus dem Einfühlungsdrange resultieren “5 und dieser Drang richte sich vor allem auf das Gefühl eines erlebten Glücks, das nur erfahren werde, indem die Annäherung an das „Organisch-Lebendige“ den Einzelnen aus „der Differenziertheit seines individuellen Bewusstseins" erlöse. ${ }^{6}$ Hier begegnet Naturalismus als höchste Form von ästhetisch vermitteltem und objektiviertem Selbstgenuss. Dabei sei dieser Genuss jedoch nur zu haben, indem die andere Seite des Kunstwollens - der Stil - die immer auch abstrahierenden Mittel zur Formgebung bereitstelle.

Bereits bei Riegl, Worringer und Dvořák ist in den Überlegungen zum Naturalismus in der Kunst eine Dimension angelegt, die der seinerseits durch die Wiener Schule geprägte Arnold Hauser - jenseits eines idealistischen Überbaus - entfalten und zuspitzen sollte: Bei ihm kommt dem Naturalismus in seiner starken Bindung an die individuelle Erfahrung und relativ unbehelligt von normativen Regeln beziehungsweise diesen entgegen das eigentlich dynamische Moment im Sinne einer Geschichte der Kunst und ihrer Fortschritte zu; während all jene gegenläufigen Tendenzen, die formalisierend und abstrahierend ausgerichtet sind, letztlich auf Erstarrung hinauslaufen würden. Für Hauser waren alle Künste in ihrem Ursprung notwendig naturalistisch, und von der magischen Funk-

3 Riegl ging es vor allem um die Darlegung intrinsischer Prozesse und Gesetzmäßigkeiten stilistischer Entwicklungen in der Kunst, entgegen dem Materialismus und der Determiniertheit der Kunst durch Technik bei Semper, aber auch als Alternative zum Idealismus oder rein psychologischen Modellen. Vgl. Alois Riegl: Naturwerk und Kunstwerk I [1901], in: ders.: Gesammelte Aufsätze, hg. von Karl M. Swoboda, Wien 1929, S. 51-64, hier bes. S. 54f. und S. 59.

4 Wilhelm Worringer: Abstraktion und Einfühlung. Ein Beitrag zur Stilpsychologie [1908], Leipzig u.a. 1981, hier v.a. S. 24f.

5 Worringer 1981 (wie Anm. 4), S. 43.

6 Worringer 1981 (wie Anm. 4), S. 30. 
tion prähistorischer Bildwerke bis zum Theater um 1900 liege hierin die Relevanz von Kunst als vitalem Teil von Kultur und Form der sozialen Auseinandersetzung. ${ }^{7}$

Keine dieser Varianten des Operierens mit unerschütterlichen Dualismen und Oppositionen erscheint heute irgendwie anschlussfähig. Dennoch deutet sich schlaglichtartig an, dass die Rede von Naturalismus verschiedene Perspektiven und Probleme implizieren kann, die weit hinausreichen über jene mehr oder weniger obsoleten Entweder-OderKonstellationen, in denen der Begriff oft verwendet wird. Diese Mehrdimensionalität gilt es aufzugreifen, wenn man sich dem Thema adäquat nähern will und sie ist für uns ein Anlass gewesen, in diesem Buch von Naturalismen im Plural zu sprechen.

Vordergründig reagiert diese Rede von Naturalismen auf den einfachen Fakt, dass der Begriff - allein im Feld von Kunst und Ästhetik - auf sehr verschiedene Art verwendet wurde und wird. So wird Naturalismus als historische Kategorie verwendet und steht zum Beispiel für charakteristische Tendenzen in Literatur und bildender Kunst, im Theater oder dem Film um 1900. Zugleich lässt sich beobachten, dass der Terminus häufig bestimmte Eigenschaften von Werken, Modi ästhetischer Erfahrung und somit eher systematische Kriterien künstlerischer Praxis bezeichnet. Dieser Frage, was Naturalismus für Musik, Literatur oder bildende Kunst überhaupt und jeweils medienspezifisch bedeuten kann, geht Martin Zenck in seinem Aufsatz nach. Dabei ist keineswegs gesagt, dass historische und systematische Perspektiven getrennt voneinander auftreten. Im Gegenteil: Häufig greifen beide Facetten kaum trennbar ineinander. Spezifische Qualitäten von Kunstwerken, die als naturalistisch charakterisiert werden, bekommen - etwa im späten 19. Jahrhundert - programmatischen Status für bestimmte Akteure und Strömungen; oder als naturalistisch verstandene Eigenschaften und Merkmale werden - wie in der Rede von ,Naturalismus der Renaissance' - zu einer der Signaturen einer ganzen Epoche. Zunächst trägt der hier verwendete Plural also dem einfachen Faktum dieser Vielgestaltigkeit Rechnung und erkennt sie als Teil des Problemzusammenhangs an, um den es uns geht.

Darüber hinaus hoffen wir mit dieser Perspektive auf die Vielzahl an Naturalismen nicht bei dem erwähnten Dualismus stehen zu bleiben. Dieser spannt sich gewöhnlich zwischen Oppositionen wie den folgenden auf: Naturalismus versus Idealismus, Naturnachahmung versus abstrakte Form, und schließlich Natur versus Kultur als menschliches Konstrukt. Es ist die anhaltende Wirkung dieser in ihrer Absolutheit längst fragwürdigen Oppositionen, die Naturalismus als Thema schlechthin zu einem verminten Gelände hat werden lassen. Wer dieses Thema aufgreift, setzt sich dem Sog dieser vermeintlichen Gegensätze aus und allein die Frage nach Naturalismen kann schnell den Verdacht erwecken, selbst - mit allen Differenzierungen - irgendwie Naturalist zu sein.

7 In dieser marxistisch geprägten Sozialgeschichte der Kunst kommt dem Naturalismus die Rolle der eigentlich bestimmenden Strömung aller Kunstgeschichte zu. Formalisierende Gegentendenzen werden als Phasen innerhalb dieser relativiert und die Anerkennung dieser Tatsache sei ein untrügliches Kriterium für die progressive - oder auch konservative - Positionierung des einzelnen Wissenschaftlers. Vgl. Arnold Hauser: Sozialgeschichte der Kunst und Literatur [1953], München 1975. 
Im Rahmen der skizzierten Dualismen würde dies aber bedeuten, die Kunst vor allem oder gar ausschließlich durch natürliche Prozesse und Faktoren determiniert zu sehen, jede Formalisierung zugunsten einer irgendwie unmittelbaren Empirie und getreuen Abbildung zu verneinen, und - besonders schwerwiegend weil direkt politisch relevant das einzelne Artefakt und alle in ihm verkörperten Sinnkonstruktionen, Wertvorstellungen oder Urteile zu naturalisieren, das heißt aus der Verantwortung menschlichen Handelns an die Natur zu delegieren beziehungsweise aus ihr abzuleiten. - Die Absurdität einer solchen Position ist evident und so kann die Frage lediglich sein, ob und wie man derartigen Spiegelfechtereien in falschen Polarisierungen entgeht und welche Perspektiven dies eröffnen kann.

Eine Option, sich dieser Vielgestaltigkeit der Naturalismen in ihren verschiedenen Facetten und Valenzen anzunehmen, liegt im Versuch einer begriffsgeschichtlichen Klärung - und hilfreiche Beiträge in diesem Sinne liegen durchaus vor. ${ }^{8}$ Sie liefern nützliche Abgrenzungen zu benachbarten Begriffen, wie etwa dem des Realismus oder Verismus. Und sie erhellen einige der Sinnprägungen und Diskurse, in denen Naturalismus explizit verhandelt wurde. Aber genau hier liegen auch die Grenzen begriffsgeschichtlicher Zugänge: Sie konzentrieren sich durchaus folgerichtig überwiegend auf solche Dokumente und Quellen, in denen Naturalismus - bezeichnenderweise im Singular - in programmatischem Sinne oder, in kunsthistorischer Perspektive, als allgemeine Stiltendenz fast immer eine Seite in einem extrem polarisierten Feld markiert. Nahezu unvermeidlich begegnet der Terminus als Kampfbegriff versus Zielscheibe der Kritik, oder er ist Teil mehr oder weniger strikt dualistisch organisierter Entwürfe von Kunst- und Ästhetikgeschichte. Genau das aber birgt und verdeckt die eigentlich relevanten Aspekte und Fragen.

Mit dem bis hierher Gesagten sind bereits wesentliche Schwierigkeiten benannt, sich dem Naturalismus-Thema adäquat zu nähern. Als Rahmung und Ausblick auf die hier zusammengestellten Beiträge seien vier unterschiedliche Problemzusammenhänge aufgezeigt, in denen naturalistische Perspektiven entweder besonders relevante historische Positionen definierten oder noch immer virulente Fragen beinhalten. Dies betrifft das Verhältnis von Natur und Kunst, die mediale Spezifik naturalistischer Qualitäten, die Normativität der Natur sowie das Verhältnis von Wahrnehmung und Erkenntnis.

8 Boris Röhrl: Kunsttheorie des Naturalismus und Realismus. Historische Entwicklung, Terminologie und Definitionen, Hildesheim u. a. 2003; Yves Chevrel: Naturalistisch, in: Karlheinz Barck u.a. (Hg.): Ästhetische Grundbegriffe. Historisches Wörterbuch, 7 Bde., Stuttgart u.a. 2000-2005, Bd. 4, S. 404-432. 


\section{Kunst als Natur - Natur als Kunst}

Die Forderung nach Naturnachahmung ist schon in der Antike ein Topos, der nicht nur für die bildenden Künste, sondern gleichermaßen auch für Poesie, Schauspielkunst und Musik bemüht wurde. Für Vitruv ist die Natur so die unübertreffliche „Lehrerin und Lehrmeisterin“.9 Alle menschlichen Erfindungen seien „,von der Schöpferkraft der Natur vorgeschaffen ${ }^{\text {" }}{ }^{10}$ Diese bedingungslose Vorbildlichkeit der Natur erhält spätestens mit der Renaissance eine neue Virulenz. Ungeachtet auch stark idealisierender Tendenzen werden die Arbeiten von Künstlern unentwegt an ihrer Ähnlichkeit zur Natur und ihren Werken gemessen. Diese Fremd- und Selbstverpflichtung der Kunst auf die Natur kann man als Naturalismus beschreiben - vor allem dann, wenn der Imperativ der Naturnachahmung zum vorrangigen oder gar einzigen Wertmaßstab avanciert.

Dabei galt als keineswegs ausgemacht, in welchem Maße Künstler dieser Forderung überhaupt gerecht werden könnten, heißt ob es sich bei dem „Stechen“ zwischen Natur und Kunst, von dem Ludovico Dolce spricht, überhaupt um einen fairen Wettstreit mit offenem Ausgang handelt. ${ }^{11}$ Einerseits nämlich galt die Natur als unerreichbar. Für Marsilio Ficino etwa ist die Natur kategorisch lebendiger, wirkkräftiger und schöner als menschliche Hervorbringungen. ${ }^{12}$ „Jeden Tag erfahren wir, dass die Natur vorzüglicher ist als die Bildkunst“, erinnert Joachim Camerarius den Leser seines Emblembuchs. ${ }^{13}$ „[A]llein in dem scheid sich die natur und der Maler vonn einander“, hält Paracelsus fest, „das die natur lebendig ding macht / der maler tode ding / die natur wesentliche ding / der maler schatend ding. ${ }^{\text {"14 }}$ Ein Wurm, hält Galileo Galilei fest, ist vollkommener als jede Statue. ${ }^{15}$ Die lebendig über den Tisch krabbelnde Fliege, konstatiert Gian Lorenzo Bernini, ist ein besseres Porträt des Papstes als jedes Gemälde. ${ }^{16}$

Andererseits mangelt es aber auch nicht an Stimmen, welche die Chancen der Kunst optimistischer einschätzen. Noch zurückhaltend argumentiert so der Maler Pozzoserato,

9 Vitruv: Zehn Bücher über Architektur, hg. von Curt Fensterbusch, Darmstadt 1964, S. 461.

10 Vitruv 1964 (wie Anm. 9), S. 461.

11 Giovanni B. Pittoni und Lodovico Dolce: Imprese di diversi Precipi, Duchi, Signori, ed altri personaggi et huomini letterati et illustri, Venedig 1562, Nr. 51.

12 Marsilio Ficino: Platonic Theology, hg. von Michael J.B. Allen u. a., 6 Bde., Cambridge u. a. 20012006, Bd. 1, S. 252 (IV.1.5): „[...] tanto vavaciore sapientioreque arte quanto efficit efficacius et efficit pulchriora."

13 Joachim Camerarius: Symbolorum et emblematum ex animalibus quadrupedibus desumtorum centuria altera collecta, Nürnberg 1595, fol. 29v, Nr. 21: „[...] Quod quidem ut in nonnullis verum esse concedimus, sicuti in arte pictoria \& similibus: ita in pluribus aliis locum habere nequit, Naturamque multo esse praestantiorem quotidie experimur."

14 Paracelsus: Von den Mineralien, in: ders.: Etliche Tractat, Straßburg 1582, S. 370-438, hier S. 395f.

15 Galileo Galilei: Dialog über die beiden hauptsächlichsten Weltsysteme, das ptolemäische und das kopernikanische, hg. von Emil Strauss, Leipzig 1891, S. 108.

16 Domenico Bernini: Vita del Cavalier Gio. Lorenzo Bernino, Rom 1713, S. 96. 
dass angesichts der Ähnlichkeit von Kunst und Natur, allein der Kunst das Lob gebühre. ${ }^{17}$ Ähnlich behauptet die Inschrift eines der Bauwerke im Park von Schloss Hellbrunn, dass dies den alten „Zweifel löse, ob Kunst über der Natur steht oder Natur über der Kunst“ ${ }^{\text {. }}{ }^{18}$ Deutlicher wird die Inschrift auf einem von Goltzius entworfenen Kupferstich, die behauptet, die „geschickte Malerei“ habe den Wettstreit mit der „mächtigen Natur“ mit „ewig siegreichen Tafeln“ für sich entschieden. ${ }^{19}$ Vergleichbar spricht Sperone Speroni davon, dass in Tizians Werken die Dinge schlicht „besser von ihm gemalt sind, als von der Natur hervorgebracht “ ${ }^{20}$ Und unverhohlen fordert Ludovico Dolce, dass ein Maler nicht nur danach jagen solle, die Natur zu imitieren, sondern sie zu übertreffen. ${ }^{21}$ Ficino schließlich pauschalisiert: „Der Mensch imitiert also alle Werke der göttlichen Natur und perfektioniert, korrigiert und verbessert die Werke der niederen Natur. ${ }^{\text {“22 }}$

Die Reihe von Beispielen ließe sich endlos fortführen. In vielen Fällen handelt es sich dabei um leere Floskeln des Lobes, in denen sich vor allem noch einmal die unhinterfragte Vorbildlichkeit der Natur manifestiert. Allerdings wurden bisweilen auch mediale und rezeptionsästhetische Differenzen aufgerufen, um das Bild der ,besseren Kunst' zu untermauern: Jacopo da Pontormo argumentierte dafür, dass die Malerei durch den Einsatz von Licht und Farbe nicht nur Dinge schaffen könne, „welche die Natur nie hervorgebracht hat“, sondern die Erzeugnisse der Natur auch „verbessert werden und durch die Kunst Schönheit erhalten“; ${ }^{23}$ Federico Borromeo rühmte Jan Brueghels d. Ä. Stilllebenkunst, da seine Blumen dauerhafter als die vergängliche Natur seien; ${ }^{24}$ und für Dolce

17 Lodovico Pozzoserrato: Epitaph, Federzeichnung, $256 \times 166$ mm, 26. Mai 1597, Amsterdam, Historisch Museum, Inv.-Nr. A.18032; die Inschrift lautet: „Si Del Par Natura | et Arta | E Nata | qual è la Magior- | la laude. Data!“

18 „Dubium, an ars sit supra naturam, vel natura supra artem, marmorea haec structura resolvet.“

19 Übersetzung nach Lubomír Konečný, in: Thomas Fusenig u.a. (Hg.): Hans von Aachen (15521615). Hofkünstler in Europa, Ausst.-Kat. (Suermondt-Ludwig-Museum Aachen u.a.), Berlin u.a. 2010, S. 175, Nr. 51; das Original lautet: „Nobile si quid humus, si quid tenet Amphitrite, | Spectatu dignum si quid olympus habet, || Æmula naturæ dextra pictura potenti | Semper uicturas transtulit in tabulas. || Sed rudis est omnis sine Pallade forma: | Si coniurarint, pulchrius his quid erit? || Et comes ambabus si uenerit inclijta uirtus, | Vndiq perfectum laurea cinget opus.“

20 Sperone Speroni: Dialogo d'amore, in: ders.: Dialogi, Venedig 1543, fol. 25v-26r: „[...] come quello, che della vita dell'huomo solamente il color della pelle ci rappresenta, et non piu oltra. TAS: Voi fatte torto à Titiano: le cui imagini sono tali, et si fatte, che eglie meglio l'essere dipinto da lui, che generato dalla natura."

21 Lodovico Dolce: Dialogo della pittura intitolato l'Aretino, Venedig 1557, fol. 29r: „Deue adunque il Pittore procacciar non solo d'imitar, ma di superar la Natura.“

22 Ficino 2001-2006 (wie Anm. 12), Bd. 4, S. 170 (XIII.3.1): „Denique homo omnia divinae naturae opera imitatur et naturae inferioris opera perficit, corrigit et emendat.“

23 Zitiert nach der Übersetzung von Kurt W. Forster: Pontormo. Monographie mit kritischem Katalog, München 1966, S. 121.

24 Wiedergegeben nach Eberhard König und Christiane Schön (Hg.): Stilleben, Bonn 1996, S. 129. 
wirkt nichts in der Natur „so anziehend und erfreut das Auge des Betrachters so sehr wie die Malerei, weder die Edelsteine noch das Gold." ${ }^{\text {25 }}$

Die schwersten Schläge gegen die Musterhaftigkeit der Natur hinwieder führten diejenigen Autoren, welche nach der Beschaffenheit der Natur selbst fragten und ausloteten, wo genau ihre künstlerische Nachahmung anzusetzen habe. Schon aus theologischer Sicht war Vorsicht geboten. Die post-lapsale Natur konnte kaum noch als vollkommene Schöpfung Gottes und bedingungsloser Maßstab für das menschliche Tun gelten. Platonisch gemünzt erschien die empirisch fassbare Welt nur als matter Abklatsch der ursprünglichen Idee. Vincenzo Danti argumentiert daher in seiner Disegno-Schrift, dass die Form aller Naturdinge zwar perfekt angelegt sei, es bei ihrer Aktualisierung in der Materie aber zu Fehlern komme. Künstler sollten daher nicht oberflächlichen Erscheinungen nacheifern (ritrarre), sondern die zugrunde liegenden göttlichen Ideen nachahmen (imitare) ${ }^{26}$ Genau in dieser Abkehr von einer naiven Mimesis visueller Daten bei gleichzeitigem Insistieren auf dem Imperativ der Naturnachahmung liegt ein Paradox, das die Wurzel des frühneuzeitlichen Naturalismus ausmacht. Wie Jan van Brevern in seinem Beitrag zeigt, kann Johann G. Sulzer in dieser Argumentationstradition dann auch Natürlichkeit zu einer dezidiert künstlichen Eigenschaft erklären, die der Natur selbst gerade nicht zukommt. In der antiken Legende von Zeuxis, der kein irdisches Vorbild zum Malen der idealschönen Helena fand und daher auf das Studium von sieben krotoniatischen Jungfrauen auswich, spiegelt sich diese Hoffnung auf eine Kunst, deren Werke die Natur übertrifft. Eng verbunden mit diesem idealistischen Naturalismus ist daher die Umwertung der Imagination zum entscheidenden künstlerischen Vermögen. ${ }^{27}$ Mittels außergewöhnlicher Phantasie seien Künstler in der Lage zwischen geistiger und materieller Welt zu vermitteln sowie die in der Natur angelegten Ideen anschaulicher zu machen, als diese selbst es vermöge. Genau in diesem Sinne behauptet Pietro Aretino, dass Tizian „in seinem Pinsel die Idee einer neuen Natur" habe und seine Kunst erst die Natur zu sich selbst führe. ${ }^{28}$ Entsprechend konstatiert Lomazzo, dass der Künstler von „der Welt geliebt und von der Natur gehasst“ worden sei. ${ }^{29}$

25 Nach Gudrun Rhein: Der Dialog über die Malerei. Lodovico Dolces Traktat und die Kunsttheorie des 16. Jahrhunderts, Köln u. a. 2008, S. 261.

26 Vincenzo Danti: Il primo libro del trattato delle perfette proporzioni di tutte le cose che imitare, e ritrarre si possano con l'arte del disegno, Florenz 1567, u.a. S. 57.

27 Vgl. dazu einführend Alain Godet: „Nun was ist die Imagination anderst als ein Sonn im Menschen." Studien zu einem Zentralbegriff des magischen Denkens, Diss. Basel 1982 sowie Marieke van den Doel: Ficino en het voorstellingsvermogen. Phantasia en imaginatio in kunst en theorie van de Renaissance, Amsterdam 2008.

28 Pietro Aretino: Lettere sull'arte, hg. von Ettore Camesasca, 4 Bde., Mailand 1957-1960, Bd. 2, S. 210, Nr. 409: „E chi sa che Tiziano (che, come dissi altre volte, ha nel pennello la idea d'una nuova natura) [...].“; ebd., Bd. 1, S. 77, Nr. 47: „Io nel vederlo chiamai in testimonio essa natura, facendole confessare che l'arte s'era conversa in lei propria."

29 Paolo Lomazzo: Trattato dell'arte della pittura, scoltura, et architettura, Mailand 1584, S. 112: „[...] era amato dal mondo \& odiato dalla natura [...].“ 
Eine verwandte, wenn gleich etwas anders geartete Begründung der Möglichkeit durch Nachahmung der Natur diese selbst zu übertreffen, entsprang der bis weit in die Frühe Neuzeit gültigen Unterscheidung zwischen einem statischen und einem dynamischen Naturbegriff. ${ }^{30}$ Kaum erreichbar sei die Natur - allein schon aufgrund der medienspezifischen Gebundenheit der Kunst - hinsichtlich ihrer ideellen Anlage (natura naturata), die sich in den äußerlichen Erscheinungen der Natur manifestiert. Die sich aktual darstellende Natur könne der Künstler nur übertreffen, wenn er die Prinzipien nachahme, nach denen die Natur selbst fortwährend schöpferisch tätig ist (natura naturans). Auch dies mündete in eine Abkehr von simpler Oberflächen-Mimesis und bedeutete einen Naturalismus, der am treffendsten als naturmagisch charakterisiert ist. Gelungene Naturnachahmung bedeutete demnach wie ein Alchemist, Werke in Analogie zur Natur hervorzubringen, wie ein Astrologe sich natürlicher Kräfte zu bedienen, oder wie ein Hieroglyphiker Naturuniversalien ins Bild zu setzen. ${ }^{31}$ Entsprechende Verklärungen frühneuzeitlicher Künstler und ihrer Werke sind zahlreich: Michelangelo wurde mit dem Statuenmagier Hermes Trismegistos gleichgesetzt, Luca Giordano portraitierte sich als Alchemist, Pieter van Laer (il Bamboccio) als Magier und unzählige Male wurden die Faszination von Bildern, der Einfluss von Künstlern oder die Alchemie der Farben beschworen. ${ }^{32}$

Die über Jahrhunderte prägende Forderung nach Naturnachahmung stellte das Kunstwerk in eine spannungsvolle Unbestimmtheit zwischen Naturalium und Artefakt. So ist die Behauptung nicht übertrieben, dass die Stärke der Analogisierung von Kunst und Natur geradezu daraus entsprang, dass paradoxerweise die künstlerische Qualität eines Werkes durch die Betonung seiner Natürlichkeit untermauert wurde. Dabei blieb vordergründig unhinterfragt, dass es sich bei Kunstwerken um Artefakte handelt. Und genauso unumstritten war es, dass die Qualitäten von Kunstwerken nicht völlig losgelöst von den Erzeugnissen der Natur erklärt werden konnten. Wenn Aristoteles feststellt, dass die menschliche techné und die Natur strukturanalog verfahren, weil ihre Produkte immer Synthesen aus Form und Stoff seien, dann ist jede menschliche (aber auch jede tierliche) Kunst immer schon eine zweite Natur. ${ }^{33}$ In diesem Sinne hat auch Victor Hugo,

30 Jan Białostocki: The Renaissance Concept of Nature and Antiquity, in: Ida E. Rubin (Hg.): The Renaissance and Mannerism, Princeton 1963 (=Acts of the $20^{\text {th }}$ International Congress of the History of Art, Bd. 2), S. 19-30.

31 Vgl. Maurice Saß: Physiologien der Bilder. Naturmagische Felder frühneuzeitlichen Verstehens von Kunst, Berlin u.a. 2016, u.a. S. 11-30.

32 Luca Giordano: Selbstportrait mit Destillierkolben, Öl/Lw., $116 \times 96 \mathrm{~cm}$, Mailand, Pinacoteca di Brera, Inv.-Nr. 598; Pieter van Laer: Selbstportrait als Magier, Öl/Lw., $80 \times 115$ cm, 1630er, Privatbesitz (The Leiden Collection, New York); Maurice Saß: „Un altro Mercurio [...] Trimegisto [...] e 'l mio buon benvenuto“. Antonio Allegrettis Beschreibung von Michelangelo und Cellini als Kinder Saturns, in: Mitteilungen des Kunsthistorischen Institutes in Florenz 58 (2016), S. 278-282.

33 Vgl. dazu einführend Arbogast Schmitt: Mimesis bei Aristoteles und in den Poetikkommentaren der Renaissance, in: Andreas Kablitz und Gerhard Neumann (Hg.): Mimesis und Simulation, Freiburg/Br. 1998, S. 17-53. 
wie Andrea Haarer in ihrem Aufsatz zeigen kann, seine nicht-figurativen Zeichnungen als Nachvollzug natürlicher Prozesse begriffen.

Die Vorstellung vollkommener Naturmimesis der Kunst und einer natürlichen Kunst kannte insofern zwei Seiten: Einerseits ließ sie sich begreifen als naturalistische Stilisierung der Natur zum Ausgangspunkt und Wesensgrund aller Kunst; andererseits eigneten sich mimetische Kunstwerke als Stillstellungen oder Fixierungen der Natur dazu, ein Verständnis von Natur als überhaupt nur künstlerisch gestaltet zu denkende Kreation zu stützen. Dosso Dossis Schmetterlinge malender Jupiter oder Aretinos Beschreibung des Abendhimmels nach Maßgabe der Kunst Tizians sind dafür zwei bekannte Beispiele. ${ }^{34}$ Viel tiefgründiger und allgemeiner noch ist die topische Vorstellung der Welt als machina oder überhaupt als Schöpfung eines dem Menschen gleichenden Gottes. Eine grundlegende Rechtfertigung des Naturalismus lag darin, dass auch Geneseprozesse der Natur nicht ohne Rückgriff auf artifizielle Fertigungsvorgänge beschrieben werden konnten. Der Naturalismus der Kunst bedeutete nicht nur den unermüdlichen Wunsch nach einer möglichst naturgleichen Kunst, sondern implizierte vor allem auch eine Sichtweise auf die Natur als näherungsweise kunstgleich. In diesem Chiasmus von Kunst als Natur und Natur als Kunst gründet die epochale Tragweite des Naturalismus, der weit über den Bereich der Kunst hinausreicht. Menschliches Tun erscheint in dieser Perspektive als immer natürlich, die harte Grenzziehung zwischen Natur und Kunst obsolet. Vielmehr wird naturalistische Kunst so zum Katalysator eines mechanistischen oder zumindest gestalteten und gestaltbaren Naturbilds. Oder anders formuliert: Das Gelingen von Kunst kann als Garant der Beherrschbarkeit der Natur dienen. „Der Mensch“, resümiert Raffaello Borghini in seinem Riposo, „hat auch, fast als wäre er die Natur selbst, deren großartigsten Werke als menschliches Werk erscheinen lassen; und zwar hat er dies mit der Bildhauerei und mit der Malerei gemacht, indem er den Himmel nachbildet. ${ }^{\text {“35 }}$

\section{Medien und Übersetzungsprozesse}

Ob im Sinne dichotomischer Konstellationen oder auch im Feld des soeben skizzierten Chiasmus von Kunst und Natur: Die Charakterisierung von Kunstwerken, Arbeitsweisen und Theorieansätzen als naturalistisch markiert ein starkes Determiniert-Sein durch Natur. Und dies impliziert häufig ein eher geringes Interesse, ja eine tendenzielle Blindheit gegenüber den spezifischen Medien, Arbeitsweisen und Übersetzungsprozessen. Naturalismus

34 Dosso Dossi: Jupiter beim Malen von Schmetterlingen, Öl/Lw., $112 \times 150 \mathrm{~cm}$, Krakau, Wawel, Inv.Nr. 9110; Pietro Aretino: Lettere, 6 Bde., Venedig 1542-1557, Bd. 3, fol. 47r-v.

35 Raffaello Borghini: Il riposo, Florenz 1584, S. 9 f.: „Ma chi vorra lasciando queste sottili considerationi con cose più sensibili conoscere l'eccellenza dell'huomo, rimiri i suoi marauigliosi effetti nel ritrouamento di tante arti, e di tante scienze [...] ha voluto etiandio, quasi fosse l'istesssa Natura, le più eccellenti opere di lei per opera humana fare apparire; e questo ha fatto con la scultura, e con la pittura: contrafacendo il Cielo [...].“ 
scheint immer schon eine vermeintliche Unmittelbarkeit zu evozieren und dabei spielen zwei Momente eng zusammen.

Da sind zum einen die ästhetischen Qualitäten konkreter Kunstwerke - hierzu zählen die vermeintlich ungefilterte Detailfülle sowie hohe Präzision der Darstellung - oft als ,Naturtreue' bezeichnet - oder etwa synästhetische Wahrnehmungen und suggestive Präsenzerfahrungen. Häufig gelten sie als Charakteristika naturalistischer Kunstwerke und haben in der Tat die Tendenz, im Als-ob des ästhetischen Scheins das Gemacht-Sein der Werke zu überspielen. Augentäuschender Illusionismus zum Beispiel ist eine besondere Form dieser scheinbaren Unmittelbarkeit, die in der Malerei in den Spielarten des Trompe-l'œil in besonderer Weise zugespitzt wie auch konterkariert und in eine reflexive Wendung überführt wurde. ${ }^{36}$ Dieses Spiel der Täuschung operiert explizit mit der scheinbaren Grenzaufhebung zwischen Kunstwerk und Natur und diese Infragestellung ontologischer Kategorien auf Seiten des Artefakts findet auch auf Seiten der Rezipienten ihre Entsprechung. Dies geschieht dann, wenn die kategoriale Unterscheidung zwischen Mensch und Tier zum Terrain möglicher Verkehrungen wird: So etwa im Wettstreit zwischen Zeuxis und Parrhasios, in dem Zeuxis - selbst ein Meister malerischer Illusion sich vom Bild seines Kontrahenten täuschen lässt, so wie zuvor die Vögel von den Trauben auf seinem eigenen Bild getäuscht wurden. ${ }^{37}$ In dieser topischen Figur wird täuschend echte Naturwiedergabe zum Balanceakt zwischen ästhetischem Effekt und Urteilsvermögen. Und dies scheint wiederum auch in allgemeinerer Hinsicht signifikant zu sein: Naturalismus in den Künsten impliziert - auch jenseits des Trompe-l'œil als Sonderfall meist eine nahezu ausschließliche Fokussierung auf wirkungsästhetische Momente (Stijn Bussels führt dies unter Rückgriff auf rhetorische Modelle eindrücklich vor Augen); und diese Akzentuierung drängt die Materialität, die Medienspezifik und konkrete künstlerische Verfahren tendenziell in den Hintergrund.

Diese Verdrängung findet zum anderen seinen direkten Reflex nicht selten dort, wo denn doch etwas über die Entstehung naturalistischer Kunstwerke gesagt wird. Hier wird häufig suggeriert, dass diese Werke nicht nur ungeachtet bestehender Traditionen und Regeln hervorgebracht würden, sondern auch unter weitgehendem Verzicht auf kompositorische Ordnung, Auswahl und andere Entscheidungen - geradezu als würde diese Kunst schlechthin in weit geringerem Maße als andere auf dem methodisch planvollen Vorgehen

36 Zum Spiel mit der Differenz von Bild und außerbildlicher Welt, Ähnlichkeit und Selbstreflexivität des Bildes als allgemein kunsttheoretischem Problem vgl. Gottfried Boehm: Die Lust am Schein im Trompe-l'oeil, in: Ortrud Westheider u. a. (Hg.): Täuschend echt. Illusion und Wirklichkeit in der Kunst, Ausst.-Kat. (Bucerius Kunst Forum, Hamburg), München 2010, S. 24-29. Auch hier spielen spezifische Medialitäten und künstlerische Verfahren vor der Moderne bezeichnenderweise kaum eine Rolle.

37 Gemeint ist jener Wettstreit zwischen Zeuxis und Parrhasios, bei dem Zeuxis Trauben so täuschend gemalt hat, dass Vögel herbeikamen um sie zu fressen, den Wettkampf aber verlor, da er einen gemalten Vorhang vor dem Bild seines Kontrahenten, als wirklichen Vorhang verkannte. Cajus Plinius Secundus d. Ä.: Naturalis historia, hg. von Roderich König und Gerhard Winkler, Düsseldorf u. a. 1997, Buch XXXV, S. 56-59 (XXXVI, § 65f.). 
eines menschlichen Subjekts beruhen. ${ }^{38}$ Stattdessen ist ein irgendwie spontaner und authentischer Ausdruck von Seiten der Sujets und Gegenstände - als Natur im weiteren Sinne - implizites Versprechen und latentes Missverständnis in der Rede von Naturalismus. ${ }^{39}$

Diese verbreitete Minimierung der Rolle künstlerischer techne wird wiederum in kontrastierender Weise dort besonders pointiert, wo die Bildgebung tatsächlich ganz oder teilweise nicht von Menschenhand erfolgte. In diesem Sinne untersucht Dominic Olariu in seinem Beitrag die frühesten erhaltenen Fälle sogenannter Naturselbstdrucke und fragt gezielt nach den gestalterischen Mitteln zur Steigerung ihrer epistemischen Werte. Die Frühe Neuzeit operierte hier mit zahlreichen Varianten der Vorstellung einer gleichsam spielerisch tätigen Natur und ihrer Hervorbringungen, den ludi naturae. ${ }^{40}-$ Und die wohl bekanntesten Beispiele für ein Echo derartiger Vorstellungen in der Moderne sind wiederum Bezeichnungen der frühen Photographie als photogenic drawing und pencil of nature, in denen die Kunst der Zeichnung als Metapher und Modell einer Selbst-Darstellung von Natur geprägt wurde. ${ }^{41}$ Philipp Müller zeichnet dies in seinem Beitrag nach und verdeutlicht zugleich die daraus hervorgehenden Komplikationen im Zeitalter digitaler Bilder.

Erst die jüngere Forschung hat die Herausforderung angenommen, eine wechselseitige Verzahnung von Natur und Naturkonzepten mit der Praxis und Theorie der Künste sowie deren Medialität, techne und Übersetzungsvorgängen auch für die Frühe Neuzeit als systematischen Zusammenhang zu untersuchen. Nur wenige Beispiele seien hier genannt, die zum direkten Hintergrund der Planungen für unsere Tagung und den vorliegenden Band gehörten: Da sind etwa Studien zur Stilllebenmalerei des 17. Jahrhunderts als Adaptation natürlicher Transformationsprozesse und Experimentierfeld zur Natur der Farben, ${ }^{42}$ Arbeiten zu Naturabguss und zeichnerischer Konstruktion als komplementären Modellen frühneuzeitlicher Konzepte natürlicher Formbildung, ${ }^{43}$ oder Forschungen zu

38 Dies trifft insbesondere für die programmatischen Entwürfe einer naturalistischen Kunst im späten 19. Jahrhundert zu; hier oft verbunden mit einem Anspruch, über die ästhetisch ungeschönte Wiedergabe von Wirklichkeit auch psychologische Situationen und soziale Milieus für eine breite Öffentlichkeit zu erfassen. Vgl. die Beiträge in Gabriel P. Weisberg (Hg.): Illusions of Reality. Naturalist Painting, Photography, Theatre and Cinema. 1875-1918, Amsterdam u.a. 2010; sowie einführend insbes. Gabriel P. Weisberg: Reframing Naturalism, in: ebd., S. 18-29.

39 Aus dieser Perspektive entstanden die Planungen einer weiteren Tagung der Forschungsstelle Naturbilder, deren Beiträge publiziert werden in: Frank Fehrenbach und Matthew Vollgraff (Hg.): Ökologien des Ausdrucks / Ecologies of Expression, Berlin u. a. (in Vorbereitung).

40 Grundlegend Paula Findlen: Jokes of Nature and Jokes of Knowledge. The Playfulness of Scientific Discourse in Early Modern Europe, in: Renaissance Quarterly 43 (1990), S. 292-328; siehe auch die Beiträge in Natascha Adamowsky, Hartmut Böhme und Robert Felfe (Hg.): Ludi naturae - Spiele der Natur in Kunst und Wissenschaft, München 2011.

41 Vgl. hierzu Peter Geimer: Von selbst entstandene Bilder - Zerstreuung der Autorschaft, in: Adamowsky, Böhme und Felfe 2011 (wie Anm. 40), S. 285-297.

42 Karin Leonhard: Bildfelder. Stilleben und Naturstücke des 17. Jahrhunderts, Berlin 2013.

43 Robert Felfe: Naturform und bildnerische Prozesse. Elemente einer Wissensgeschichte in der Kunst des 16. und 17. Jahrhunderts, Berlin u. a. 2015. 
Magie und Astrologie als Komponenten einer Physiologie der Bilder in der Renaissance. ${ }^{44}$ Die Forschungsstelle Naturbilder / Images of Nature hat sich im ersten Jahr ihrer gemeinsamen Arbeit derartigen Konstellationen unter dem Aspekt von Kräften in Natur, Ästhetik und Künsten gewidmet, und dabei den historischen Horizont auf die Moderne erweitert. $^{45}$

Fragen und Perspektiven wie die hier angedeuteten verkörpern sich exemplarisch in einer Radierung von Robert de Chastillion, die erstmals in den 1670er Jahren in Paris als Teil einer Histoire des Plantes gedruckt wurde (Abb. 1). ${ }^{46}$ Das Buch gab sich zugleich als wissenschaftliches Gemeinschaftswerk der noch jungen Académie Royale des Sciences und wollte auch den erfahrenen Graphikliebhaber ästhetisch ansprechen. Mit suggestiver Finesse geben die Graphiken - wie hier, im Falle zweier Moose - den filigranen Bau und die Beschaffenheit der Oberflächen einschließlich virtuoser Lichtwirkungen wieder. Minutiös werden sich wiederholende Formen dabei nahe an eine Übersetzung in reine Struktur herangeführt - und doch bleibt die Integrität des Pflanzenkörpers gewahrt. Auf diese Weise substituieren die Drucke ihre Objekte in Bildkörpern von erstaunlicher suggestiver Präsenz und insistieren auf deren individuellen Erscheinungen. In diesem Fall widersetzte sich diese Akzentuierung gezielt den Interessen und Methoden der Taxonomie, die im Sinne ihrer Arbeit an immer feineren Ordnungskriterien auf formalisierende Darstellungskonventionen setzte. Entgegen dieser dominierenden Tendenz in der zeitgenössischen Botanik setzten die Autoren und Graphiker dieses Buches auf eine Physiognomie der Pflanzen, die versuchte noch im Druck die irreduzible Fülle sinnlicher Erfahrungen zu transportieren.

Zoomt man sich in die Feinstruktur dieser Graphiken hinein (vgl. Cover-Abb.) - und dazu fordern sie den Leser immer wieder heraus - vollzieht sich zwischen Bild und Betrachter ein weiterer Twist: Die einzelnen Lineaturen der Radierung erschließen die pflanzlichen Formen wie die räumlich gestaffelten Schichten eines höchst filigranen, artifiziellen Gewebes. Dabei erstasten sie dessen Formen und Strukturen, als wären sie als graphische Spur selbst vor allem eine Funktion des einfallenden Lichts und somit Effekt der natürlichen Situation. Es kommt so zu einer doppelten Anverwandlung zwischen Natur-Ding und Bild, die so nur im Intaglio-Druck und seinen aufs äußerste verfeinerten Linientechniken initiiert werden kann. Der damit aufgeworfenen Frage nach der medialen Gebundenheit naturalistischer Qualitäten geht Karin Leonhard in ihrem Beitrag zu Dürers Hasen nach und zeigt, wie dessen malerischen Nachahmungen den Naturalismus ihres Vorbilds transformierten.

Saß 2016 (wie Anm. 31).

Frank Fehrenbach, Robert Felfe und Karin Leonhard (Hg.): Kraft, Intensität, Energie. Zur Dynamik der Kunst, Berlin u.a. 2018.

Hier nach erneutem Abdruck in Denis Dodart: Receuil des plantes gravées par ordre du Rio Louis XIV, Paris 1786-1788, Taf. 236; hergestellt und erstmals gedruckt in Denis Dodart: Mémoire pour servir d'Histoire des Plantes, Paris 1676. 


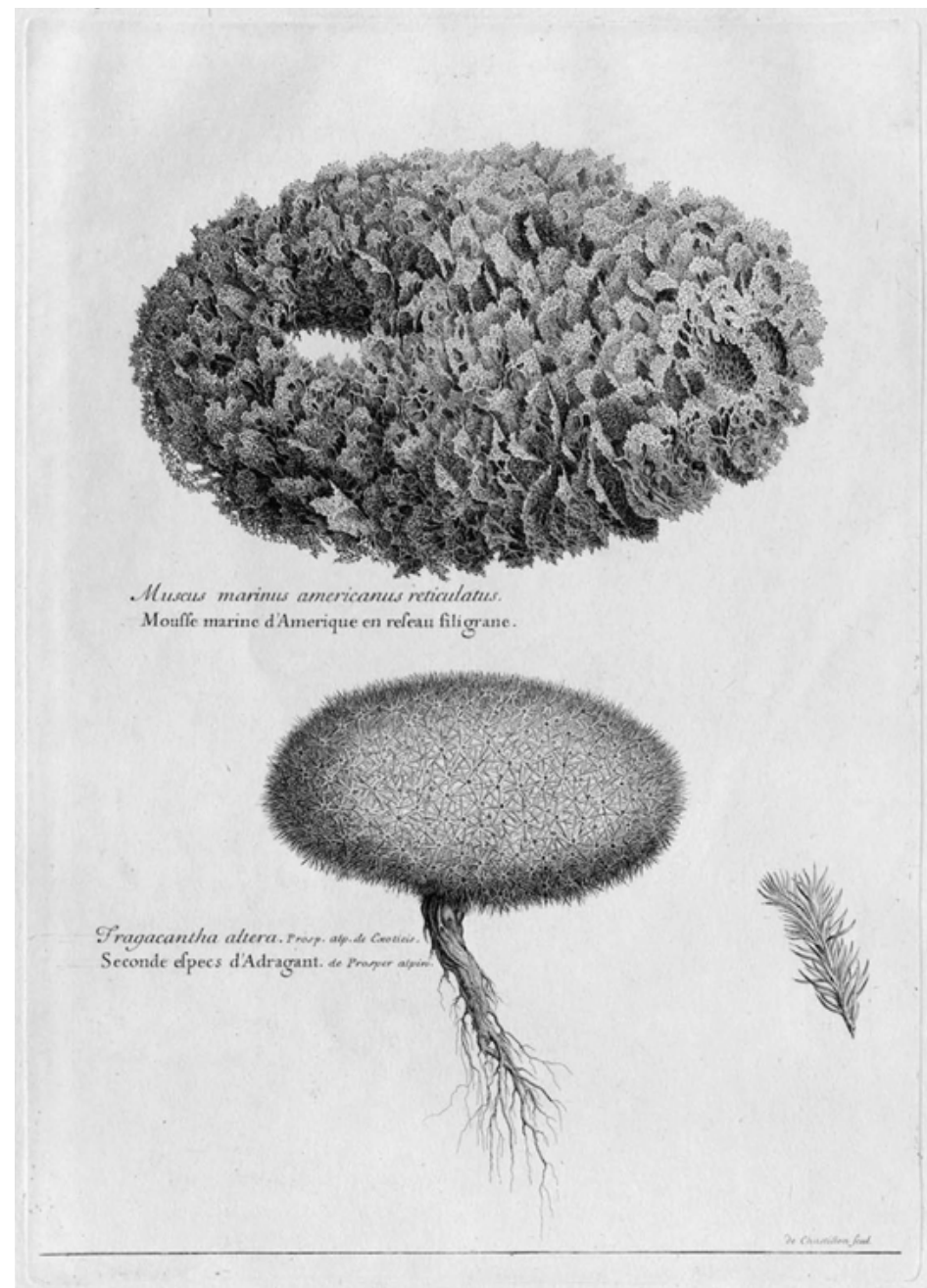

1 Muscus marinus americanus reticulatus, in: Denis Dodart: Receuil des plantes gravées, Paris 1676/1788

Wir sind sehr froh, für unsere Tagung und für den Band Esther Kinsky als komplementäre Stimme zu den Aufsätzen aus verschiedenen Wissenschaftsdisziplinen gewonnen zu haben. Ihre Romane und ihre Lyrik erkunden die spannungsreiche Kontinuität von Naturerfahrung als konkrete Situation und Grundierung für fast alle Facetten persönlichen Lebens wie auch für die gegenwärtig massiven Verschiebungen etwa in urbanen Umgebungen. Die beharrliche Näherung an Möglichkeiten, diese Momente von Natur sprachlich zu artikulieren, ist ein besonders markanter poetischer Impuls ihres Schreibens. 


\section{Normierungsprozesse}

Das Verhältnis von Mensch und Natur liegt quer zum gängigen Chiasmus philosophischer Traditionen, da höchst volatil ist, was in dieser Doppelperspektive auf den Menschen und die ihn umgebende Welt mit dem Begriff, Natur' überhaupt bezeichnet ist: die menschenleere Wildnis, die Grundlage menschlicher Ernährung, das Ökosystem, in dem der Mensch lebt, oder die natürlich gegebene Beschaffenheit des Menschen selbst in körperlicher, kognitiver, emotionaler oder geistiger Hinsicht. Natur ist ein kulturgeschichtliches Produkt. Dieser Einwand ist ernst zu nehmen und nicht einfach als sprachphilosophischer Dekonstruktivismus abzutun. Janina Wellmann zeigt in ihrem Beitrag auf, wie sehr das heute in den Natur- und Lebenswissenschaften gültige Bild der Natur weder von der einen Natur ausgeht noch überhaupt diese als ein statisches Fixum entwirft, sondern als höchst artifizielle Modellierung spezifischer Beobachtungskontexte begreift. Gerade diese Unschärfe oder Dehnbarkeit des Naturbegriffs hat seine argumentative Einbindung in die unterschiedlichsten normativen Diskurse ermöglicht. Natur gehört zu den reflexartigen Fixpunkten der Legitimierung von etwa ästhetischen und moralischen Maximen. Dabei ist es für die Plausibilität naturalistischer Ableitungen unerheblich, ob Natur als unerreichbares Ideal gesetzt wird oder als rohe Wildnis und geistloser Urzustand die negative Talsohle bildet, über die sich der Mensch und seine Werke erheben sollen.

Dieses Beziehen auf Natur ist denkbar allgemein. Sinnlich anschauliche Dinge bilden als vermeintlich schnell akzeptierte Gegebenheiten vielleicht überhaupt die Grundlage von Verständigung und Gemeinschaftsgefühl. Die ganze Sprache lässt sich als System erstarrter Metaphern verstehen, dem der zeigende Gestus auf, da draußen' (in die Natur) zugrunde liegt. Die ältesten Bilder und Mythen erzählen davon. Unzählige Höhlenmalereien werden von Tieren bestimmt. Das Gilgamesch Epos beginnt mit dem Sehen aller Dinge. Und am Anfang der Bibel steht ein Schöpfungsbericht. Natur ist die übliche Karte menschlicher Standortbestimmung.

Christlich gemünzt entspricht dies der Rede von der Natur als zweitem Buch der göttlichen Offenbarung. In der Natur wird der göttliche Wille anschaulich, zeigt sich sein Wesen und die Schönheit der Schöpfung ist nur Ausdruck der göttlichen Güte. Allerdings ist diesem christlichen Verständnis nach die Natur eben auch nur zweitrangig und eben nur materieller Abklatsch unendlich vollkommenerer Werte des Himmels - insbesondere in ihrem gefallenen Zustand nach dem Sündenfall. Natur ist nur soweit von Bedeutung, wie in ihr Geistiges erkennbar ist. Für einen aufgeklärten Leser ist es deswegen ein Leichtes, die Naturbeschreibung etwa des Physiologus als Folge einer christlichen Sichtweise zu lesen, und es ist ihm fast unmöglich, in dessen Darstellung unterschiedlicher Tiere etwas anderes als die christliche Projektion geistiger Werte zu sehen. Die Natur selbst ist hier nicht unbedingte moralische Instanz, sondern nur Medium. Naturalistisch hingegen wird die Orientierung in oder an der Natur, wenn sie absolut gesetzt und zur Letztbegründung menschlichen Tuns oder Sollens gemacht wird. 
Diese Wende lässt sich ikonographiegeschichtlich gut fassen: Im Mittelalter wurde die Natur gewöhnlich als Schmiedin dargestellt, die im Auftrage Gottes fortwährend die irdischen Schöpfungen zum Leben erwecke. Auf zahlreichen Minitaturen sieht man sie als zuverlässige Arbeiterin, auf deren Amboss Menschen und andere Tiere gestaltet werden. Dieses Bild der Natur als handwerkliche Dienerin Gottes, die himmlische Formen in irdische Materie bringt, verschwindet mit der Renaissance quasi vollständig. Stattdessen etablieren sich zwei Formen der Natur-Personifikation: zum einen als nackte Frau, die mit der Milch ihrer Brüste die Natur nährt; zum anderen nach Maßgabe der antiken Göttin Diana Ephesia als Standbild, in deren Ornament sich die Ordnung der Natur spiegelt. In beiden Fällen tritt die Natur als autonome Instanz und als aus sich wirksames Prinzip auf. Natur ist nicht länger die Handlangerin Gottes, sondern selbst eine Göttin. Dieser Wandel lässt sich als Naturalismus beschreiben, insofern die Natur verabsolutiert und als Grund eigenen Rechts gesetzt wird. ${ }^{47}$

Die Vorbildlichkeit der Natur für das Arbeiten des Künstlers und die Bewertung seiner Werke ist in dieser Hinsicht nur eine Facette eines viel grundsätzlicheren Phänomens menschlicher Kultur. Genaugenommen ist es schwer, überhaupt einen Bereich menschlichen Lebens zu benennen, für das die Natur im Ganzen oder einzelne natürliche Erscheinungen nicht als Maßstab herangezogen worden sind. Zahllos sind die Verweise auf die natürliche Beschaffenheit des menschlichen Körpers, um geschlechtliche Rollenbilder zu legitimieren. Legion sind die Referenzen auf das Leben von Tieren, um ein normatives Idealbild der menschlichen Familie zu stützten. Und topisch sind die Rückgriffe auf Gesetzmäßigkeiten in der Natur, um verschiedenste politische Formen des gesellschaftlichen Zusammenlebens zu untermauern. Die Darstellung zweier Inuits auf einem aus Augsburg stammenden Flugblatt, dessen moralischen und theologischen Implikationen Christopher Heuer in seinem Beitrag aufzeigt, bildet in dieser Hinsicht ein anschauliches Beispiel. Ein anderes liefert der frühneuzeitliche Sternenglaube mit seinen naturalistischen Erklärungen individueller Schicksale oder größerer Zusammenhänge. Abgelöst wurde er unter anderem von Klimatheorien, die spätestens mit Winckelmann auch in direktem Bezug zur kulturellen Produktion gebracht wurden und noch im 19. Jahrhundert in der Zusammenschau mit dem sozialen Milieu-Begriff hoch brisant blieben, wie Michael F. Zimmermann in seinem Aufsatz zeigt. Die Vergöttlichung natürlicher Kräfte und Gesetzmäßigkeiten von sogenannten Naturvölkern ist strukturell nicht verschieden vom neuzeitlichen Rückgriff auf die Natur zur diskursiven Etablierung von ,natürlichen'

47 Vgl. dazu einführend Wolfgang Kemp: Natura. Ikonographische Studien zur Geschichte und Verbreitung einer Allegorie, Frankfurt/M. 1973; Mechthild Modersohn: Natura als Göttin im Mittelalter. Ikonographische Studien zu Darstellungen der personifizierten Natur, Berlin 1997; zu dem mittels dieses Naturverständnis legitimierten Geschlechterverständnis vgl. Londa Schiebinger: Am Busen der Natur. Erkenntnis und Geschlecht in den Anfängen der Wissenschaft, Stuttgart 1995. 
Verhaltensnormen. Hierin liegt eine der Pointen von Bruno Latours kritischer Feststellung, wir seien nie modern gewesen. ${ }^{48}$

Die europäische Aufklärung ist ohne naturalistische Positionen kaum zu denken. Und gleichzeitig ist der Naturalismus ein Erbe der christlichen Tradition. Natur im Universalsingular ist das aufklärerische Substitut für eine Schöpfung, an deren Spitze Gottvater steht. Spätestens Autoren wie John Stuart Mill, Thomas Henry Huxley oder Émile Zola haben es deswegen zu einem intellektuellen Gemeinplatz werden lassen, in der Natur keine Werte zu sehen, sondern nur das empirische Faktum, das ununterbrochen der mannigfaltigen Deutungsmaschinerie menschlicher Kultur unterworfen ist. ${ }^{49}$ Seit George Edward Moore 1903 seine Principia ethica veröffentlichte wird die Ableitung von Werten und Imperativen von als gesetzt und natürlich verstandenen Gegebenheiten als eine Form von naturalistischem Fehlschluss bezeichnet. Und mit David Hume wird die argumentative Wendung von Ist-Zuständen in moralische Maximen als Sein-Sollen-Fehlschluss entkräftet. Es ist ein Unterschied, ob man annimmt, dass der Mensch den Dynamiken und Gesetzen der Natur unterliegt (sei es der Planeten oder der Schwerkraft), oder ob man diese zu Imperativen erhebt. Die Aussage, Man soll der Schwerkraft gehorchen', macht für uns heute keinen Sinn, weil man die Schwerkraft für ein unumstößliche Naturgesetz hält. Die gesellschaftliche Akzeptanz von Eigenschaften und Kräften der Natur ist der Schleier des naturalistischen Fehlschlusses.

Mit dieser Fortschrittsgeschichte ist das Bild aber noch nicht vollständig: Lorraine Daston hat jüngst dafür argumentiert, dass die scharfe Wende gegen naturalistische Fehlschlüsse als philosophische Position nicht absolut zu setzen ist und in ihrer Zuspitzung vielmehr eine historische Besonderheit der letzten hundert Jahre darstellt, die ihrerseits höchst aufschlussreich hinsichtlich des rezenten Selbstverständnisses wissenschaftlicher und politischer Kulturen ist. ${ }^{50}$ Schlichtweg gegen naturalistische Kulturtheorien und Ethiken zu sein, heißt auch den Mensch nicht primär in Abhängigkeit zur Natur und der ihn umgebenden Welt zu denken, das heißt eine Dichotomie von Natur und Mensch, Kultur oder Geist zu postulieren. Wohin dies führen kann, stellen die großen Herausforderungen gegenwärtiger Naturzerstörungen vor Augen. Die philosophische Dekonstruktion der dialektischen Spannung von Kultur und Natur kann auch Naturentfernung und Allmachtsphantasien Tür und Tor öffnen. Wert dezidiert nicht naturalistisch zu stützen, sondern metaphysisch zu begründen, kann in religiöser oder ideologischer Diskriminierung münden. Nicht ohne Grund finden sich in der jüngsten Vergangenheit die feurigsten Verfechter naturalistischer Positionen im Bereich der Human Animal Studies und des Ecocriticism.

48 Bruno Latour: Wir sind nie modern gewesen. Versuch einer symmetrischen Anthropologie, Berlin 1995.

49 Vgl. einführend den kritischen wie historisch einordnenden Aufsatz von Larraine Daston: The Naturalistic Fallacy is Modern, in: Isis 105 (2014), S. 579-587.

50 Daston 2014 (wie Anm. 49). 
Die in diesem Band versammelten Aufsätze beleuchten das vexierbildhafte Verhältnis von Kunst und Natur, das heißt sie analysieren Naturalismus binokular als Ideal oder Trugschluss beziehungsweise Natur als Vorbild und Gegenbild menschlichen Tuns - und zwar nicht nur im Falle der stupenden Mimesis einzelner Kunstwerke, sondern als kulturtheoretisches Problem, welches das Verständnis von so unterschiedlichen Kategorien wie Kunst, Wissenschaft und Ästhetik über Jahrhunderte ko-präfigurierte und noch heute methodische Weichenstellung kulturhistorischen und kunstwissenschaftlichen Arbeitens ist: Kultur als Sprössling der Natur; Natur als humanoides oder allemal tief in der europäischen Kulturgeschichte verankertes Konstrukt.

\section{Wahrnehmung und Erkenntnis}

Spätestens seit der Frühen Neuzeit kommt ein weiterer Strang hinzu. Gemeint sind die vielfältigen Allianzen, die sich nun zwischen der Herstellung und dem Gebrauch von Bildern und einer wissenschaftlichen Erforschung der Natur nachweisen lassen. Von der Zeichnung als Methode zum Studium sichtbarer Phänomene wie auch hypothetischen Denkens bis zur druckgraphischen Bildausstattung von Publikationen gingen bildliche Darstellungen dabei sehr enge und zugleich tief gestaffelte Beziehungen zu naturwissenschaftlichem Wissen, dessen Methoden und Wahrheitsansprüchen ein. In zahlreichen Studien ist inzwischen gezeigt worden, dass der Kunst und den Bildern dabei keineswegs nur eine dienende Rolle zukam - in dem Sinne, dass sie ein vorgängiges Wissen über Natur irgendwie anschaulich vermittelt hätten. ${ }^{51}$ Vielmehr erhielten die rasanten Wandlungen und der neue Status, den die Naturwissenschaften in den folgenden Jahrhunderten erlangten, aus Bildern und Praktiken der Visualisierung wesentliche Impulse.

Schon früh haben kunsthistorische Studien nicht nur die epistemischen Qualitäten von Kunstwerken betont, sondern in diesem Zusammenhang den Terminus des Naturalismus aufgegriffen. Ebenso prominente wie wenig überraschende Beispiele hierfür sind etwa Klassiker der Forschung zu Albrecht Dürer. So bemühte sich Heinrich Wölfflin eine ausgewogene Balance zu finden, wenn er Dürers allgemeine stilistische Entwicklung einerseits - vor allem in dessen theoretischem Bemühen um Verallgemeinerung und Regeln seiner Kunst - deutlich von jenem älteren Naturalismus absetzt, der jene Kunst charakterisiere, mit der der junge Dürer aufgewachsen war. Andererseits aber hob er den Naturalismus seines akribischen Studiums natürlicher Dinge als ein zeitlebens prägendes Moment hervor. In diesem Zusammenhang weist Wölfflin denn auch Émile Zola zurück, dessen Verständnis von Naturalismus, ein zeitloses, immer gleiches Sehen implizieren würde. Der Kunstgeschichte hingegen müsse es gerade um die letztlich über-individuelle Geschichtlichkeit des Sehens gehen. ${ }^{52}$

51 Einen Überblick über Tendenzen und Probleme in der jüngeren Forschung gibt Alexander Marr: Knowing Images, in: Renaissance Quarterly 69 (2016), S. 1000-1019.

52 Heinrich Wölfflin: Die Kunst Albrecht Dürers [1905], München 2000, S. 279-282. 
Erwin Panofsky wiederum wird gerade die Synthese von einem methodischen Studium der Natur und der Systematisierung künstlerischer Praxis in Dürers Werk als „rationalen Naturalismus“ im Sinne einer intellektuellen Haltung des Künstlers charakterisieren. ${ }^{53}$ Vergleichbar hatte bereits 1927 Ernst Kris - allerdings in Hinblick auf die so genannte Dürer-Renaissance um 1600 - insbesondere dem Zeichner und Miniaturmaler Joris Hoefnagel einen explizit „wissenschaftlichen Naturalismus“ bescheinigt. ${ }^{54}$ Für Kris zeichnete sich dieser Naturalismus durch die Kombination einer feinteilig suggestiven Wiedergabe der Erscheinungen mit einem dezidierten Interesse an der Systematik der Natur ab. Die Ordnung der vier Elemente, als frühes taxonomisches Konzept, wie auch allegorische Sinnschichten fügten sich dabei als Teile in eine umfassende humanistische Gelehrsamkeit, die auch die breiten empirischen Interessen der Medizin und verschiedener Felder der Naturgeschichte spiegelte.

Für die genannten Autoren und Texte ist signifikant, dass Naturalismus hier nicht primär im Sinne einer kategorialen Bestimmung und stilistisch definitiven Zuschreibung verwendet wird. Der Terminus markiert vielmehr ein Feld von starken Beziehungen zwischen künstlerischer Arbeit, Natur und Wissen als Untersuchungsgegenstand; und mit dieser Akzentuierung gingen Wölfflin, Kris und Panofsky - mit jeweils eigenen Forschungsinteressen - weit hinaus über jede nach wie vor gebräuchliche Rede von Naturalismus, lediglich im Sinne von ,naturgetreuer Wiedergabe'. ${ }^{55}$

Wichtige Impulse zur Untersuchung jeweils spezifischer Konstellationen zwischen Natur, Kunst und Wissen kamen in der zweiten Hälfte des 20. Jahrhunderts vielfach aus der englischsprachigen Forschung. So wurde etwa die Frage aufgeworfen, inwiefern naturalism in der Wissensgeschichte der Frühen Neuzeit und der naturalism der Künste, tatsächlich miteinander verbunden sind - und wenn ja, beide sich dennoch grundsätzlich voneinander unterscheiden. ${ }^{56}$ So wenig die Frage nach diesem Verhältnis pauschal - etwa mit Hinweis auf einen neuen Empirismus - zu beantworten ist, so markant sind die seit der Renaissance zunehmend systematisch reflektierten Beziehungen zwischen Bildern und Bildkonzepten zu sinnlicher Erfahrung und deren Rolle im Zusammenhang von

53 Erwin Panofsky: Das Leben und die Kunst Albrecht Dürers [1943], Hamburg 1995, S. 373.

54 Ernst Kris: Georg Hoefnagel und der wissenschaftliche Naturalismus, in: Arpad Weixlgärtner und Leo Planscig (Hg.): Festschrift für Julius von Schlosser zum 60. Geburtstag, Wien 1927, S. 243-253; hier nach dem erneuten Abdruck des Textes in: Bettina Uppenkamp (Hg.): Erstarrte Lebendigkeit. Ernst Kris - Zwei Untersuchungen, Berlin 2012, S. 11-25, hier v. a. S. $24 \mathrm{f}$.

55 Um am Beispiel Dürers zu bleiben, sei hier verwiesen auf die bis heute grundlegende Publikation zu dessen Tier und Pflanzenstudien von Karl Koreny: Albrecht Dürer und die Tier und Pflanzenstudien der Renaissance, München 1985, hier v. a. in allgemeiner Hinsicht S. 13-15.

56 James S. Ackerman: Science and Visual Art, in: Hedley Howell Rhys (Hg.): Seventeenth Century Science and the Arts, Princeton 1961, S.63-90; ders.: Early Renaissance ,Naturalism' and Scientific Illustration, in: Allan Ellenius (Hg.): The Natural Sciences and the Arts. Aspects of Interaction from the Renaissance to the 20th Century, Stockholm 1985, S. 1-15. 
Urteilsfähigkeit und Erkenntnis dargestellt worden. ${ }^{57}$ Insbesondere seit den 1990er Jahren wurden vor diesem Hintergrund in zahlreichen Fallstudien jeweils konkrete Gefüge der wechselseitigen Verschränkungen von künstlerischer Praxis, Bildtheorie, Erkenntnismethoden und Wissen untersucht. Arbeiten zu Leonardo da Vinci, ${ }^{58}$ Galileo Galilei ${ }^{59}$ oder zur Publikationspraxis in einzelnen Wissensgebieten wie der Botanik und Anatomie sind nur wenige Beispiele hierfür. ${ }^{60}$ Dabei ist in der Frühen Neuzeit der Anspruch auf Erkenntnisgewinn und Wissen über Natur keineswegs per se säkular und disziplinär fest definiert. Vielmehr konnten sich gerade an naturalistischen Qualitäten von Bildern neben Erkenntnisvermittlung auch religiöse Kontemplation und Erinnerungspraxis sowie heilsgeschichtliche Hermeneutiken von Natur festmachen. ${ }^{61}$

Eines der zentralen Probleme in dem hier lediglich skizzierten Forschungsfeld ist die Frage nach dem epistemologischen Status von Bildern: Wie und auf welche Weise konnten und können Bilder und Kunstwerke originäre Einsichten artikulieren, Erkenntnisse (mit) hervorbringen, Wissen adäquat vermitteln beziehungsweise reflexive Prozesse in Gang setzen? Worauf können sich Wahrheitsansprüche in Hinblick auf bildliche Darstellungen stützen und unter welchen Bedingungen werden sie geteilt, finden Anerkennung? Und warum können Bilder überhaupt Ebene und Medium der konträren Auseinandersetzung werden? ${ }^{62}$ - Diese Fragen sind keineswegs hinreichend beantwortet, mit dem bloßen Konstatieren eines historisch neuartigen Erklärungsanspruchs in Hinblick auf Natur, den künstlerische Arbeit seit der Frühen Neuzeit für sich in Anspruch genommen habe. ${ }^{63}$

57 Einschlägig hierzu David Summers: The Judgment of Sense. Renaissance Naturalism and the Rise of Aesthetics, Cambridge 1987.

58 Vgl. Frank Fehrenbach: Licht und Wasser. Zur Dynamik naturphilosophischer Leitbilder im Werk Leonardo da Vincis, Tübingen 1997. Leonardos Anspruch, in der zeichnerischen Analyse von Naturerscheinungen auch deren wirkende Ursachen sichtbar zu machen, wird explizit unter dem Begriff naturalism verhandelt in: Martin Kemp: Leonardo and the Idea of Naturalism: Leonardo's Hypernaturalism, in: Andrea Bayer (Hg.): Painters of Reality. The Legacy of Leonardo and Caravaggio in Lombardy, Ausst.-Kat. (Metropolitan Museum, New York), New Haven u.a. 2004, S. $65-73$.

59 Horst Bredekamp: Galileo Galileo der Künstler. Der Mond. Die Sonne. Die Hand, Berlin 2007; Lucia Tongiorgi Tomasi (Hg.): Il cannocchiale e il pennello. Nuova scienza e nuova arte nell'etá di Galileo, Ausst.-Kat. (Palazzo Blu, Pisa), Florenz u. a. 2009.

60 Sachiko Kusukawa: Picturing the Book of Nature. Image Text and Argument in Sixteenth-Century Human Anatomy and Medical Botany, Chicago u. a. 2012.

61 In Hinblick auf naturalism in der Miniaturmalerei zwischen 15. Jahrhundert und um $1600 \mathrm{vgl}$. Thomas DaCosta Kaufmann: The Mastery of Nature. Aspects of Art, Science and Humanism in the Renaissance, Princeton 1993, als Problemaufriss hier insbesondere S.3-10; in Hinblick auf Buchgraphik im Umfeld der Physikotheologie um 1700 vgl.: Robert Felfe: Naturgeschichte als kunstvolle Synthese. Physikotheologie und Bildpraxis bei Johann Jakob Scheuchzer, Berlin 2003.

62 Einen Aufriss des Problems gibt David Topper: Towards an Epistemology of Scientific Illustration, in: Brian S. Baigie (Hg.): Picturing Knowledge. Historical and Philosophical Problems Concerning the Use of Art in Science, Toronto u. a. 1996, S. 215-249.

63 So etwa erneut in Hinblick auf den naturalism Dürers in: Larry Silver und Pamela H. Smith: Splendor in the Grass. The Powers of Nature and Art in the Age of Dürer, in: Pamela H. Smith und 
Weitaus spezifischer ist hier zum Beispiel der Vorschlag, Künstlern eine spezifische artisanal epistemology zuzuschreiben, ein spezifisches Erkenntnisvermögen, das in der eigenen körperlich-praktischen Arbeit und dem Umgang mit Materialien begründet gewesen sei und das im Naturalismus, als ästhetischer Qualität von Bildern, seinen evidenten Ausdruck gefunden habe. ${ }^{64}$ Ein solcher Zugang lässt sich argumentativ verdichten, wenn naturalistische Eigenschaften von Bildern mit spezifischen Erkenntnisinteressen und Methoden des Wissens zusammengedacht werden. So ist etwa für die Botanik das Paradigma einer morphological description als epistemologischer Grund für eine starke Position bildlicher Darstellungen herausgearbeitet worden, insbesondere für Bilder, die ihre Gegenstände in akribisch-detailreichen und suggestiv vergegenwärtigenden Aufzeichnungen wiederzugeben vermochten. ${ }^{65}$ Dieser Akzent auf der Form-Beschreibung als zentralem Erkenntnismoment in den Wissenschaften schärft denn auch den Blick auf die historische Dynamik in Status und Wertschätzung naturalistischer Bilder. Neben deren Konjunkturen lassen sich nun auch die (unvermeidlichen) Krisen und Bedeutungsverluste verstehen. So ist für die Zeit um 1600 ein Geltungsverlust naturalistischer Bildmodi im Sinne wissenschaftlicher Evidenzerzeugung verzeichnet worden; in dem Moment nämlich, in dem sich das Wissen - vor allem das große Projekt taxonomischer Ordnungen - zunehmend auf strukturelle, vom einzelnen Gegenstand abstrahierbare Merkmale richtete, die am effektivsten begrifflich und in Tabellen zu visualisieren waren. ${ }^{66}$ Georg Töpfer zeichnet dies in seinem Beitrag an einigen paradigmatischen Fällen nach. In der hier konstatierten gegenläufigen historischen Tendenz, in der naturalistische Ästhetiken von Bildern ihren Wert für das Wissen weitgehend verloren haben, zeichnen sich denn auch die Konturen für eine generelle Skepsis ab, die den möglichen Status von Bildern als epistemic images - Bilder die in besonderer Weise Gegenstand, Instrument und visuelle Verkörperung von Wissen sind - geradezu definitiv an anti-naturalistische Eigenschaften bindet. Bildern, denen eine in diesem Sinne starke Position zukommt, werden dadurch

Paula Findlen (Hg.): Merchants \& Marvels. Commerce, Science, and Art in Early Modern Europe, New York u. a. 2002, S. 29-62, hier v. a. S. 46f.

64 Pamela H. Smith: The Body of the Artisan. Art and Experience in the Scientific Revolution, Chicago u. a. 2004, hier v.a. S.8-24, 59-80 und 114-127; aufgegriffen und unter dem Schlüsselbegriff discernemnt differenziert wird dieser Ansatz z.B. in den Beiträgen in Sven Dupré und Christine Göttler (Hg.): Knowledge and Discernment in the Early Modern Arts, London u.a. 2017.

65 Claudia Swan: From Blowfish to Flower Still Life Paintings. Classification and its Images, in: Smith und Findlen 2002 (wie Anm. 63), S. 109-136. Die Episteme einer morphological description wurde hier unter anderem in Anlehnung an Svetlana Alpers: Die Kunst der Beschreibung. Holländische Malerei des 17. Jahrhunderts [1983] herausgearbeitet, ein Buch, das seinerseits einschlägige Studien zur Geschichte der Botanik inspiriert hat, wie etwa Brian W. Ogilvie: The Science of Describing. Natural History and the Renaissance Europe, Chicago u. a. 2006.

66 Vgl. Swan 2002 (wie Anm. 65); sowie am Beispiel der Zeichnungskonvolute des museo cartaceo von Cassiano dal Pozzo David Freedberg: The Eye of the Lynx. Galileo, his Friends, and the Beginnings of Modern Natural History, Chicago u. a. 2002, hier v. a. S. 3-8 und 397-416. 
charakterisiert, dass sie primär „creations of the mind's eye“ seien. ${ }^{67}$ Das heißt: Bei aller möglichen Ähnlichkeit zu dem was sie darstellen, sind sie rein gedanklichen Operationen nachgeordnet, werden durch methodische Aspekte, Kriterien und Argumente präfiguriert, die für das jeweilige Wissen relevant sind. Dieser Problemstellung, die gerade technische Bilder betrifft, geht Isabella Augart in ihrem Beitrag an einem relativ frühen Fall in Form von Philipp Apians Landkarten nach, deren naturalistischen Bildwerte sich gerade nicht in einem einfachen Abbildcharakter erschöpften, sondern auf medienspezifischen Abstraktionsprozessen fußten.

Die Publikation geht auf das dreitägige Symposium Naturalismen. Kunst, Wissenschaft und Ästhetik im Warburg Haus und an der Universität Hamburg zurück, das im Januar 2017 als dritte Jahrestagung der Forschungsstelle Naturbilder / Images of nature stattfand und von uns gemeinsam mit Frank Fehrenbach organisiert wurde. Für den reibungslosen Ablauf sind wir erneut vor allem Sue Ryall sowie Eva Landmann und den studentischen Hilfskräften Katharina Herrmann, Antonia Götz und Sanja Hilscher verpflichtet. Wir danken den Teilnehmern dieser Veranstaltung für ausgesprochen lebhafte Diskussionen. Gregory Bryda und Matthew Vollgraff übernahmen generös die Übersetzung der Abstracts ins Englische. Ohne den unermüdlichen Einsatz und die Sorgfalt von Sanja Hilscher bei der redaktionellen Arbeit am Manuskript hätte dieser Band noch lange auf seine Vollendung warten müssen. Katja Richter, Anja Weisenseel und Anna Louisa Schmidt vom Verlag De Gruyter begleiteten den Band ebenso geduldig wie beharrlich. Erneut war es ein Vergnügen, mit Petra Florath zusammenarbeiten zu dürfen. Seine Veröffentlichung wie bereits die vorangegangene Tagung ermöglichte die großzügige Unterstützung der Alexander von Humboldt-Stiftung.

Für die Mühen aller, die an dem Band mitgewirkt haben, möchten wir uns herzlich bedanken.

67 Lorraine Daston: Epistemic Images, in: Alina Payne (Hg.): Vision and its Instruments. Art Science, and Technology in Early Modern Europe, University Park/PA 2015, S. 13-35, hier S. 18. Exemplarisch angewandt wird dieses Konzept von epistemic images z.B. in Sachiko Kusukawa: Drawing as an Instrument of Knowledge. The Case of Conrad Gessner, in: ebd., S. 36-47, sowie: Kusukawa (wie Anm. 60), S. 162-177. 
